\title{
Human 3D Motion Computation from a Varying Number of Cameras
}

\author{
Magnus Burenius, Josephine Sullivan, Stefan Carlsson, and Kjartan Halvorsen* \\ KTH CSC/CVAP, S-100 44 Stockholm, Sweden \\ http://www.csc.kth.se/cvap
}

\begin{abstract}
This paper focuses on how the accuracy of marker-less human motion capture is affected by the number of camera views used. Specifically, we compare the 3D reconstructions calculated from single and multiple cameras. We perform our experiments on data consisting of video from multiple cameras synchronized with ground truth $3 \mathrm{D}$ motion, obtained from a motion capture session with a professional footballer. The error is compared for the $3 \mathrm{D}$ reconstructions, of diverse motions, estimated using the manually located image joint positions from one, two or three cameras. We also present a new bundle adjustment procedure using regression splines to impose weak prior assumptions about human motion, temporal smoothness and joint angle limits, on the $3 \mathrm{D}$ reconstruction. The results show that even under close to ideal circumstances the monocular $3 \mathrm{D}$ reconstructions contain visual artifacts not present in the multiple view case, indicating accurate and efficient marker-less human motion capture requires multiple cameras.
\end{abstract}

Keywords: Motion Capture, 3D Reconstruction, Monocular, Bundle Adjustment, Regression Splines.

\section{Introduction}

This paper addresses the challenging computer vision problem of precise markerless human motion capture from video sequences. It focuses on measuring its feasibility and achievable accuracy with respect to the number of cameras used. This is of interest to anyone who wants to acquire the accurate $3 \mathrm{D}$ motion of people performing interesting actions from real video footage. These questions have been partially explored for the standard actions of walking and jogging using the HumanEva dataset [6]. It, however, has primarily been used to compare the accuracy of reconstruction algorithms as opposed to investigating if and when computer vision can be used for accurate human motion capture.

Our motivation is that we want to reconstruct, in real time, the $3 \mathrm{D}$ motion of a football player during a real game to augment the video broadcast and enhance the viewer's experience. Presently, the most effective and reliable way to achieve this would be to map image measurements to $3 \mathrm{D}$ motions [7]. However, learning

^ Royal Institute of Technology, School of Technology and Health, Stockholm, Sweden.

A. Heyden and F. Kahl (Eds.): SCIA 2011, LNCS 6688, pp. 24-35, 2011.

(C) Springer-Verlag Berlin Heidelberg 2011 
such mappings, as they are poor at extrapolation, requires a large amount of relevant training data and unfortunately large databases of football motion capture data are not available. And perhaps, even more pertinently, the complete repertoire of football actions cannot be replicated in a motion capture studio. Thus we need to create training data from footage of players in real games.

In the absence of significant training data, there are two distinct approaches taken. One is based on tracking: given the $3 \mathrm{D}$ pose in the first frame tracking is used to compute the most likely poses for the subsequent frames [1] using either Kalman or particle filters 13 . The initialization is typically done manually and visual features of the images are matched to those hypothesized by the state of the $3 \mathrm{D}$ model. These methods can produce nice results, especially with multi-camera footage, however, they are slow and brittle and potentially require manual re-initialization when applied to fast extended motions.

The other approach is the direct geometrical solution that can be used if the image positions of the joints are known and the skeleton is assumed to be a kinematic chain 1 2 2 3 4 45. These algorithms can be adopted to both monocular and multi-camera data, although the monocular case is a decidedly more difficult. This type of approach is appealing as it is simple and fast to implement and there is a large scope for (semi-)automating the localization of the joints in the images and depth estimation. However, it is unclear if the precision of their reconstructions, even with manually clicked image joint positions, is good enough especially from monocular footage.

In this paper we explore what is the achievable accuracy for these methods given manually estimated joint locations and known camera calibrations. Thereby we estimate a lower bound for any future automated process. We also compare the accuracy of monocular reconstructions with stereo and three-view reconstructions [12. Our experiments rely on a data set consisting of video from multiple cameras synchronized with ground truth 3D motion. The motions are football actions performed by a professional player in a motion capture studio. We also present a new bundle adjustment procedure for imposing temporal smoothness, link length and joint angle constraints on the $3 \mathrm{D}$ reconstruction. This algorithm imposes the weakest possible priors on the resulting reconstruction and reduces the errors due to the noise in the estimated joint positions. The results indicate that even under close to ideal circumstances the monocular 3D reconstructions show visual artifacts not present in the multiple view case. While the latter are visually identical to the ground truth. Thus, precise reconstructions for large amounts of data is not feasible from monocular data.

The rest of the paper is organized as follows. Section 2 describes how to create an initial 3D reconstruction from measurements of image joint positions. Both the monocular and multiple camera case is mentioned. To reduce the effect of measurement noise we impose weak prior constraints on the reconstruction in section 3. Section 4 describes the new data set of motion capture synchronized with video recordings. The data set is used to test the new algorithm and the accuracy of reconstructions from varying number of cameras. In section [5] we discuss our conclusions and possible future work. 


\section{Initial 3D Reconstruction}

The human is modeled as a kinematic chain consisting of joints and links. We assume that we are given image measurements, from one or multiple calibrated cameras, of the human joint positions shown in figure 1 for all time frames. These may have been computed fully automatically, or completely manually, or somewhere in between. The joints are chosen as rotation centers to have a well defined position. Two auxiliary joints that are not rotation centers, neck and pelvis, are added so that all joints form a kinematic chain. These two joints are not measured explicitly but computed from the measurements of the other joints. The neck is defined to be the mean of the shoulders. The pelvis is defined to be the mean of the hips. Note that we do not include the hands, feet or head in our model. These body parts are not well represented by one rigid link. Their 3D reconstruction could be obtained by alternative methods, e.g. they could have standard offsets relative the other joints, or be reconstructed by a volumetric model. Given the image positions of the joints their position in 3D can be reconstructed. Two particular scenarios are examined.

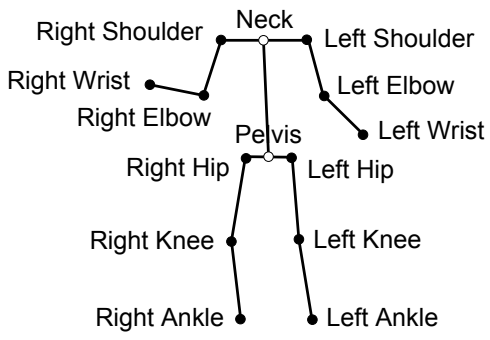

Fig. 1. Joint positions

Multiple Camera Case. When considering the joint positions from several cameras, triangulation is used to give initial reconstruction of the points in $3 \mathrm{D}$ [12. Each point at each frame is reconstructed independently of the other points. The points are not treated as a kinematic chain but as a simple point cloud.

Monocular Camera Case. In contrast when we consider measurements from just one camera the reconstruction process, due to the depth ambiguities, is more involved. The reconstruction method implemented, following previous work in this area 112 |3 4/5, makes several assumptions. These are that the skeleton is a kinematic chain with known link lengths, the depth of the root joint (required for a projective camera) and the flip of each link is known. The flip tells which end of a link that is closest to the camera. These quantities can be estimated automatically, though with difficulty [5]. This further degrades the accuracy of the obtained reconstructions is not considered within this paper. In summary, the skeletal kinematic chain is reconstructed in $3 \mathrm{D}$, by starting at the root joint and reconstructing each link in turn. 


\section{Imposing Weak Priors on the Initial Reconstruction}

Due to measurement noise the initial reconstruction might not look sufficiently good. This is especially true for monocular reconstruction. Figure 2 shows examples of measured image joint positions and the corresponding monocular 3D reconstructions from another view. Even though the measurements seems to be close to the ground truth in the camera view, the $3 \mathrm{D}$ reconstruction typically differs more if viewed from another angle. Also since the initial reconstruction is done independently for each frame it is not guaranteed to be continuous over time. An example of this is shown in the third and fourth examples of figure 2 , which displays the reconstruction of two consecutive frames.

To improve the initially estimated reconstruction we need a stronger model of what we are reconstructing. By relying more on a prior model the influence of measurement noise can be reduced. We are trying to reconstruct a human performing some arbitrary action. Therefore we do not want to restrict the prior to just model specific actions. The model should fit (almost) all possible human motions. It is just the completely unnatural motions that should be constrained. In section 3.1 we describe how to impose link length and joint angle constraints. In fact the link length constraints are already fulfilled by the initial monocular reconstruction but it should be imposed on the multiple view reconstruction as well. In section 3.2 we describe how to also impose temporal smoothness.

\subsection{Link Length and Joint Angle Constraints}

As a first step constraints can be imposed on the link lengths and joint angles to reduce the effect of measurement noise. The joints of the human skeleton cannot rotate freely. For instance the knee and elbow angles have a range smaller than $180^{\circ}$. This is one thing that makes the initial monocular estimation look weird. If these types of constraints are not imposed the arms and legs may be twisted in unnatural ways. These are the constraints typically applied in human reconstruction methods. They are used by e.g. 115], although they do not mention the details of their parametrization.
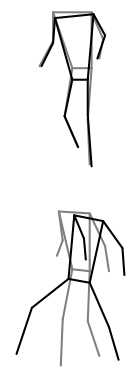
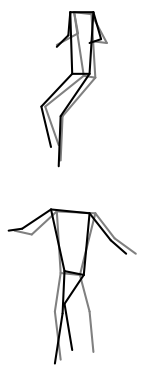
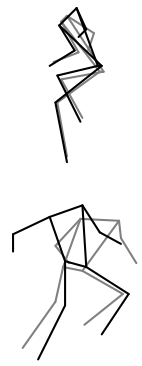
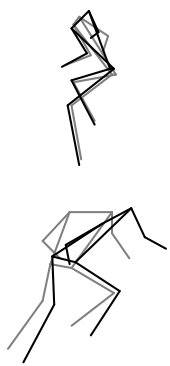

Fig. 2. The top row shows image measurements of the pose for different frames. The measurements are black and the ground truth is grey. The bottom row shows the corresponding initial monocular 3D reconstructions viewed from another direction. The reconstruction errors are significantly larger in this new view. 
To implement the constraints we let a human pose be defined by a set of joint angles and the Cartesian position of one root joint which we define to be the pelvis. The rotation of the root is not constrained and has three degrees of freedom (DOF). The elbows and knees are modeled with a single rotation DOF with upper and lower bounds. The rotation of left and right hip and shoulder have three DOF, but they should be constrained to not allow the arms and legs to be twisted unnaturally. We use the twist-swing parametrization [9]10] to deal with this and constrain the twist parameter with to be within $[-\pi / 2, \pi / 2]$. The swing component is unconstrained. This is a good approximation of the true constraints for humans 910 .

These are the parameters that are used to define a pose: 6 for the root, 3 for each shoulder and hip, and 1 for each elbow and knee, giving a total of $A=22$ parameters. This is a reduced set of parameters compared to using three Cartesian coordinates for each joint giving a total of 36 parameters. More formally let $a=\left(a_{1}, a_{2}, \ldots, a_{A}\right)$ be the generalized joint coordinates. The constraints are then expressed by linear vector inequalities, where the elements are infinite for the unconstrained components:

$$
a_{\min } \leq a \leq a_{\max }
$$

The length of all links are assumed to be known and fixed. The fixed link length constraints are fulfilled by construction in this parametrization. Let $r=$ $\left(r_{1}, r_{2}, \ldots, r_{J}\right)$ where $r_{j}$ is the homogeneous coordinates of the position of joint $j$. Denote the mapping from joint angles to the position of the joints in homogeneous coordinates as $f$ :

$$
r=f(a)
$$

A Bundle Adjustment Implementation. We now formulate an optimization problem that can be solved iteratively to get a refined estimate by imposing constraints on joint angles and link lengths. The initial reconstruction of section 2 is used as an initial guess and the joint angles $a$ are optimized to minimize the reprojection error:

$$
\begin{array}{r}
\min _{a} \sum_{c=1}^{C} \sum_{j=1}^{J} d\left(M_{c} r_{j}, z_{c, j}\right)^{2} \\
\text { s.t. } \quad a_{\min } \leq a \leq a_{\max } \\
r=f(a)
\end{array}
$$

where $C$ is the number of cameras and $J$ is the number of joints. $M_{c}$ is the projection matrix of camera $c$ and $M_{c} r_{j}$ is thus the reconstructed joint position $r_{j}$ reprojected to the image of camera $c . z_{c, j}$ are the measured image position in camera $c$ of joint $j$ in homogeneous coordinates. $d\left(p_{1}, p_{2}\right)$ is the geometric image distance between the homogeneous points $p_{1}$ and $p_{2}$ and $d\left(M_{c} r_{j}, z_{c, j}\right)$ is thus the reprojection error. The angles are constrained by inequalities (1) and the link length constraints are fulfilled by construction (equation 2). Given the initially estimated reconstruction the local minimum of the constrained minimization problem gives the refined reconstruction. The constrained nonlinear 
least squares problem is solved iteratively using the trust-region-reflective algorithm [171819], which is implemented by the MATLAB function 1sqnonlin. Note that this refinement step works the same way no matter how many cameras we use.

\subsection{Temporal Smoothness, Link Length and Joint Angle Constraints}

Both the initial estimate and the refined estimate, imposing link length and joint angle constraints, have so far been computed independently for each frame. Due to this the reconstructed animation can look jittery and unnatural, since it is not forced to be continuous. This is especially true for the monocular reconstruction. The reasonable assumption of finite muscle forces implies finite joint accelerations and continuous velocities. In this section we describe how to also impose constraints on temporal smoothness.

Interpolation. A standard way to impose temporal continuity on the reconstruction is to use interpolation. Some frames are selected as key-frames and the reconstruction is interpolated between them. Often the joint angles are interpolated linearly, using SLERP [8] for the joints having three rotational DOF. However, using linear interpolation the joint velocities will not be continuous at the key-frames and the reconstruction will look a bit stiff. To get a smoother reconstruction splines can be used [16]. We use natural cubic splines to do this leading to continuity in the second derivative at all key-frames/knots (figure 3). One drawback of interpolation is that the values at the knots are fixed. We interpolate between them but do not try to improve the values at the knots. If we have measurements taken between the knots, then those are not taken into account.

Regression Splines. The drawbacks of interpolation can be fixed by the use of smoothing splines, e.g. regression splines [16]. The idea is to let the values at the knots be variables of an optimization problem and find the ones that gives a spline that minimizes the difference to all measurements. In this way the spline that best fit the measurements can be computed (figure 3). We use equidistantly distributed knots. The smoothness is then controlled by a single parameter which is the distance between knots. The larger distance the smoother estimate. The measurements of all time frames are considered simultaneously, in a batch procedure, as opposed to standard Kalman or particle filters [13] which proceeds chronologically.

New Bundle Adjustment Algorithm. We formulate a new algorithm to perform 3D reconstruction while imposing temporal smoothness in addition to the constraints on link lengths and joint angles. The reprojection error is minimized while imposing our prior model. The difference is that now we express each angle parameter $i$ as a function of time $t$ by a regression spline:

$$
a_{i, t}=P\left(t, \alpha_{i}\right)
$$

where $a_{i, t}$ is the interpolated joint angle at time $t$ given its values at all knots $\alpha_{i}$. Let the values for all angles and knots be stored in $\alpha=\left(\alpha_{1}, \alpha_{2}, \ldots, \alpha_{A}\right)$. 

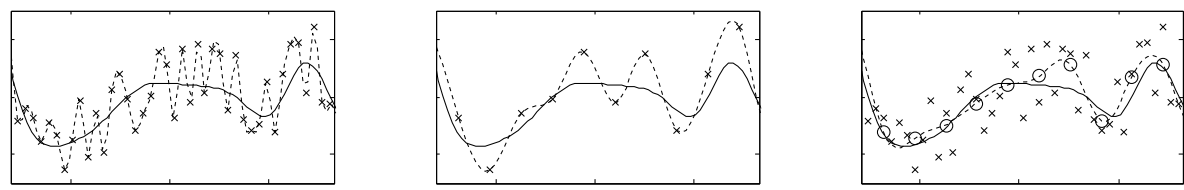

Fig. 3. The difference between interpolation and regression splines. The full line is the ground truth, the crosses are the measurements and the dashed line is the estimation. The left image shows interpolation between all measurements. The center image shows interpolation between every fourth measurement. The right image shows regression splines with a knot at every fourth measurement. Knots are drawn as circles.

The distance between knots is a variable the user may choose manually and control the smoothness of the reconstruction. It is considered to be fixed in the optimization. The initial value of all parameters at the knots are taken from the initially estimated reconstruction, described in section 2 . The values at the knots are then optimized to minimize the reprojection error:

$$
\begin{array}{r}
\min _{\alpha} \sum_{c=1}^{C} \sum_{j=1}^{J} \sum_{t=1}^{T} d\left(M_{c} r_{j, t}, z_{c, j, t}\right)^{2} \\
\text { s.t. } \begin{array}{r}
\text { min } \\
a_{i, t}=\alpha \leq \alpha_{\max } \\
r_{t}=f\left(a_{t}\right)
\end{array}
\end{array}
$$

In contrast to the previous bundle adjustment in section 3.1 all frames $(T)$ are considered simultaneously. The variables we optimize over are the joint angle parameters at all knots. Given the initial reconstructions at all knots, the local minimum to the constrained minimization problem gives the refined reconstruction. The solution is found iteratively just as in section 3.1

\section{Results}

To evaluate the accuracy of the monocular and multiple view reconstructions we obtained 3D motions of a professional footballer, using a commercial off the shelf motion capture system with passive markers. It provided the ground truth at a frequency of $200 \mathrm{~Hz}$. We used 35 markers which were not placed at the joint positions (figure 1), but from the markers the rotation centers defining the joint positions could be computed. The player was also recorded at $25 \mathrm{~Hz}$ by three regular video cameras at a resolution of 1920x1080 pixels (figure 4). A total of 30 sequences of different football actions were recorded (17 minutes of data). The videos were calibrated and synchronized to the ground truth.

Using these videos the joint image positions in the three cameras were estimated manually for seven different action instances: receiving and passing the ball, receiving ball on chest and passing, free kick, pass, defending, running plus a sharp change of direction, a contested jumping header. This corresponds in total to $16 \mathrm{~s}$. Note that since the markers used by the motion capture system 


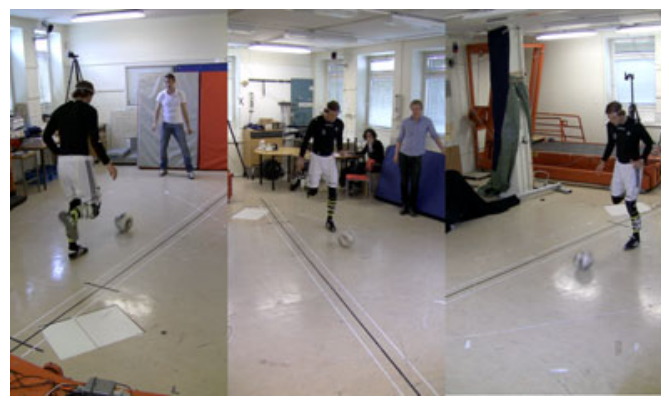

Fig. 4. A motion capture studio was used to capture ground truth animations corresponding to video from three cameras

did not correspond to the joints, the markers were not used to aid the manual joint estimation in the videos. This is important since the algorithm is intended to be used in situations outside the motion capture studio without the use of markers. These measurements were used to reconstruct the actions in 3D and the results were compared to the ground truth provided by the motion capture system. Each reconstruction was calculated using the four different algorithms described in section $2 \& 3$.

1. Initial Estimate. Independent reconstruction for each frame.

2. Limb Length \& Joint Angle Constraints. Initial estimate refined independently for each frame.

3. Limb Length \& Joint Angle Constraints Interpolated. Reconstruction (2) is computed for each key-frame and interpolated in-between.

4. Limb Length, Joint Angle \& Temporal Smoothness Constraints. Initial estimate refined by the new bundle adjustment algorithm, using regression splines in a batch process.

Note that the algorithms work on both monocular and multiple view reconstruction. Each algorithm was tested on several different camera combinations. For the initial monocular reconstruction we assumed known link lengths, flips and the depth of the root joint. This was obtained from the ground truth. Also note that the calibrated cameras were static and that the videos had little motion blur and no occlusion by any other objects. These assumptions and the fact that we did manual joint measurements in high resolution images makes it possible to compute a lower error bound under ideal conditions. In a real situation outside the motion capture studio the reconstruction will be worse.

Evaluation of the algorithms and comparison between monocular and multiple view reconstruction requires a quantitative measure of the reconstruction error. This measure should correspond to the perceived error. The most disconcerting part of a reconstruction is if the joint angles are unnatural. We therefore look at the mean joint angle error:

$$
\frac{1}{T A} \sum_{t=1}^{T} \sum_{i=1}^{A}\left|a_{i, t}-\hat{a}_{i, t}\right|
$$


However, it might not be appropriate to combine the angles describing 1D rotations and the ones describing 3D rotations by the twist-swing decomposition. For this reason we also define an error measure that only takes the mean over the 1D angles, describing the knees and elbows.
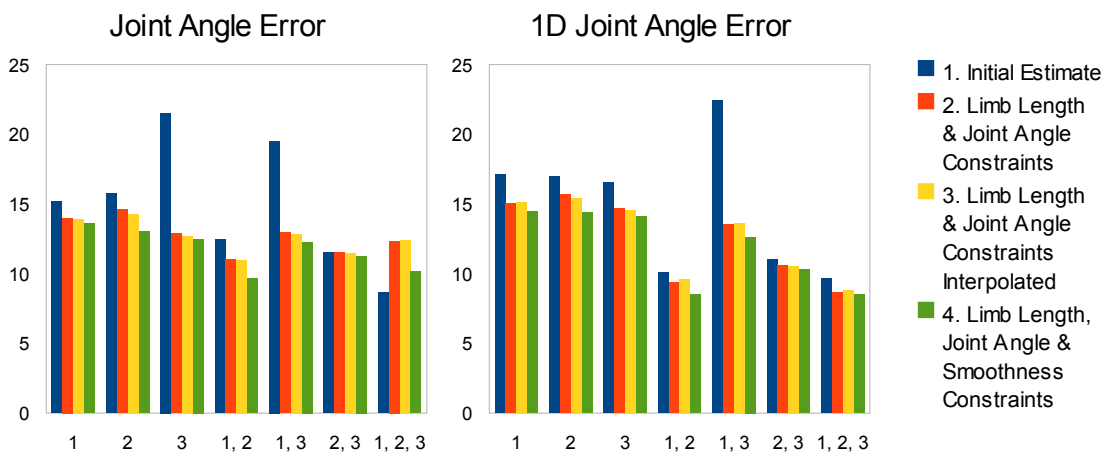

Fig. 5. The mean reconstruction error in degrees, vertical axis, resulting from different camera configurations, horizontal axis. Four different methods of reconstruction are evaluated. The errors are the mean taken over seven different motions with a total duration of $16 \mathrm{~s}$.

Figure 5] show these mean reconstruction errors over all mesured sequences, for different algorithms and camera configurations. The 1D joint angle error shows a big difference between the monocular and multiple view cases. The error is almost twice as large for the monocular case. As expected the initial estimate (1) has the largest error and our new algorithm based on regression splines (4) has the lowest, while algorithm (2) and (3) are somewhere in-between. However, except the initial estimate the difference between the algorithms are not that big in these measures, especially not between algorithm (2) and (3). Nevertheless, in the monocular case the smooth reconstructions produced by algorithms (3) and (4) looks much nicer than the jittery reconstructions produced by (1) and(2). In this sense the perceived error is not captured well by these error measures. One would likely need an error measure involving derivative approximations to capture this aspect. Disregarding the nice appearance of temporal smoothness the new algorithm (4) still performs best even with this simple error measure.

Figure 6 shows monocular and stereo reconstructions compared to the ground truth for four different frames (one per quadrant). The reconstructions is viewed from each camera. In this figure camera 1 is used for monocular reconstruction and camera 1 and 2 for stereo reconstruction. In the view of camera 2 it can clearly be seen that the monocular reconstruction does not give the same accuracy as the stereo reconstruction. The two frames at the bottom are consecutive and show that the initial monocular reconstruction is not consistent over time.

Looking at the reconstructions in motion our subjective opinion is that for the three camera case the initial estimate always looks good. For our measurement accuracy the bundle adjustments does not give a visible improvement in this 


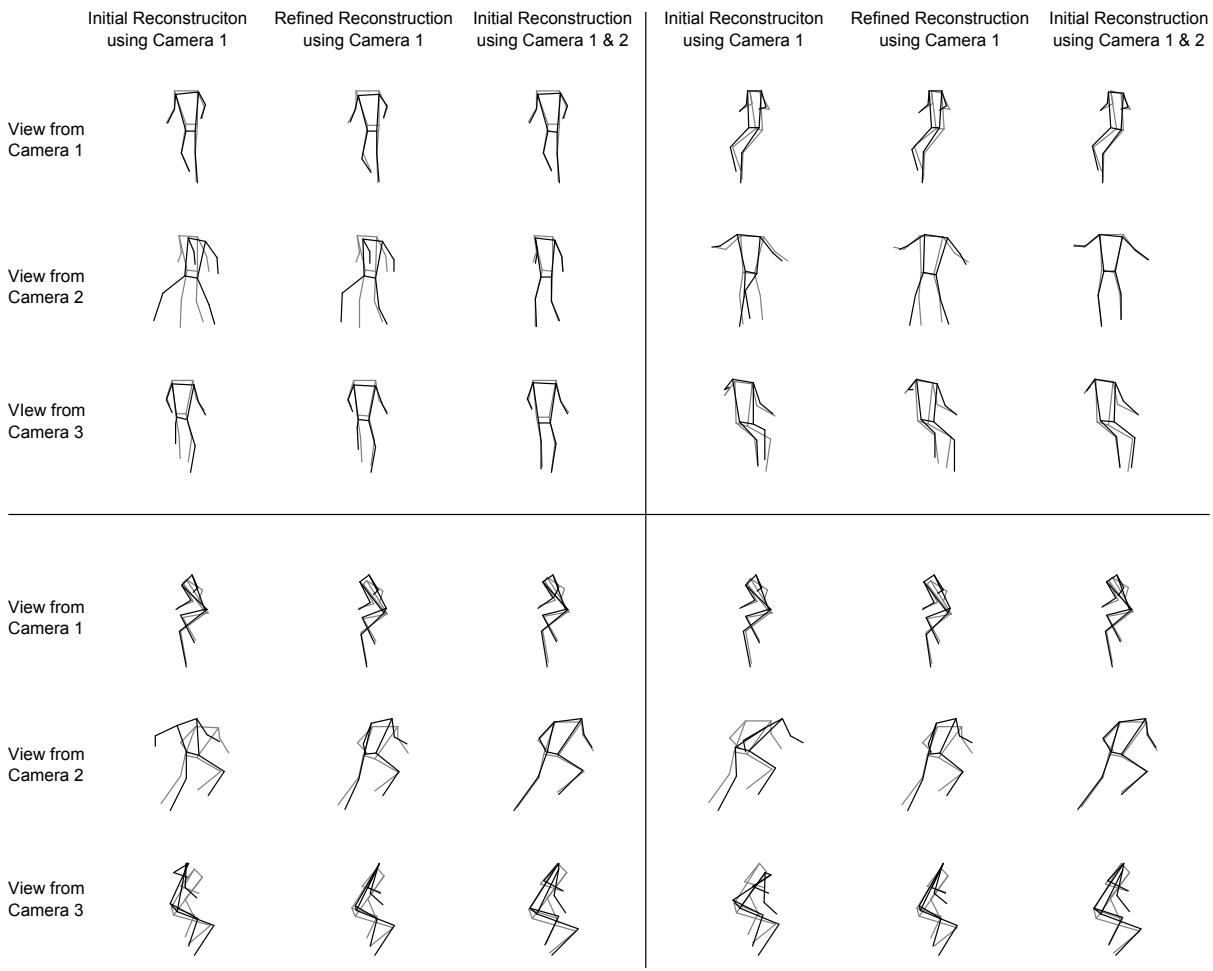

Fig. 6. Each quadrant shows the $3 \mathrm{D}$ reconstruction (black) and the ground truth (gray) for a single frame. The first column shows the initial monocular $3 \mathrm{D}$ reconstruction obtained from camera 1 . The second column shows the refined monocular reconstruction, imposing constraints on joint angles and temporal smoothness. The third column shows the stereo reconstruction obtained from camera $1 \& 2$. Each row shows the reconstructions in one of the three cameras. The view from camera 2 is the interesting one since the monocular reconstruction looks significantly worse than the stereo reconstruction from this view. Camera 3 is almost facing camera 1 and for this reason both reconstructions look all right in it, although the stereo reconstruction looks slightly better.

case. For the two camera case the initial estimate mostly looks good. It looks a little bit wobbly sometimes. In those cases the reconstruction by algorithm (4) gives a small but visible improvement. The reconstruction from camera $1 \& 3$ looks much worse than the reconstructions from camera $1 \& 2$ or camera $2 \& 3$. This is since camera $1 \& 3$ are almost facing each other. For the bad reconstructions from camera $1 \& 3$ algorithm (4) gives a big improvement. This improved reconstruction looks about the same as the reconstructions from camera 1 \& 2 and camera $2 \& 3$. In the monocular case the initial estimate generally looks bad. It does not improve that much by just imposing the joint angle constraints of algorithm (2). Imposing the smoothness of algorithm (3) or (4) gives a bigger improvement. The reconstruction of algorithm (4) looks slightly better than that of (3). However, even if this refined reconstruction looks much better than the 
initial estimate there is still a visible difference compared to the ground truth, or to the multiple view reconstructions. This is in accordance with the quantitative data shown in figure 5 and the 1D joint angle error which shows a big difference between the monocular case and the multiple view.

\section{Conclusion}

By imposing constraints on the link lengths, joint angles, and temporal continuity, the refined estimate created by the bundle adjustment algorithm generally looks better then the initial estimate. However, in the monocular case the improvement is not big enough. Even under close to ideal conditions the monocular reconstruction looks significantly worse than the multiple view reconstruction. For our final application of reconstructing 3D motion from a real football game, we can expect even worse accuracy. To make the monocular reconstruction look good it is necessary to manually tweak the measurements until the 3D pose looks correct. However, then the 3D reconstruction is not really created from the measurements of the image joint positions, but rather constructed manually in this feedback loop. If two or three cameras were used both the initial and refined estimate look similar to the ground truth. In the two camera case the initial estimate looks bad for a small set of frames, but those are improved in the refinement step.

Future Work. We conclude that monocular 3D reconstruction does not give the accuracy our application requires. The next step is then to try to use multiple cameras to accurately reconstruct the 3D motion of a player, outside the motion capture studio. For our football application we would like to deal with multiple uncalibrated rotating and zooming cameras. Affine factorization [14] and auto-calibration 15 can then be used to get the initial reconstruction and the bundle adjustment algorithm could be extended to also impose smoothness on the camera calibration. It would also be of interest to explore to what extent the joint localization can be automatized given the accuracy requirements.

\section{Acknowledgment}

This work was supported by the EU project "Free-viewpoint Immersive Networked Experience" and The Swedish Foundation for Strategic Research and the Knowledge Foundation in the project "Large Scale Visualization of Human Action". The authors would also like to thank Tracab for arranging the motion capture session and Per Karlsson (AIK) for being the subject in our experiments.

\section{References}

1. Lee, H.J., Chen, Z.: Determination of 3D human body postures from a single view. Computer Vision, Graphics, and Image Processing 30(2), 148-168 (1985) ISSN 0734-189X, doi:10.1016/0734-189X(85)90094-5 
2. Taylor, C.J.: Reconstruction of articulated objects from point correspondences in a single uncalibrated image. In: Proceedings of IEEE Conference on Computer Vision and Pattern Recognition, vol. 1, pp. 677-684 (2000)

3. Eriksson, M., Carlsson, S.: Monocular reconstruction of human motion by qualitative selection. In: Proceedings of Sixth IEEE International Conference on Automatic Face and Gesture Recognition, May 17-19, pp. 863-868 (2004)

4. Parameswaran, V.: View independent human body pose estimation from a single perspective image. In: CVPR (2004)

5. Wei, X.K.: Jinxiang Chai: Modeling 3D human poses from uncalibrated monocular images. In: 2009 IEEE 12th International Conference on Computer Vision, September 29-October 2, pp. 1873-1880 (2009)

6. Sigal, L., Balan, A., Black, M.: HumanEva: Synchronized Video and Motion Capture Dataset and Baseline Algorithm for Evaluation of Articulated Human Motion. International Journal of Computer Vision 87(1), 427 (2010)

7. Bo, L., Sminchisescu, C., Kanaujia, A., Metaxas, D.: Fast Algorithms for Large Scale Conditional 3D Prediction. In: IEEE International Conference on Computer Vision and Pattern Recognition (June 2008)

8. Shoemake, K.: Animating rotation with quaternion curves. SIGGRAPH (1985)

9. Baerlocher, P., Boulic, R.: Parametrization and Range of Motion of the Ball-andSocket Joint (2000)

10. Grassia, F.S.: Practical parameterization of rotations using the exponential map. Journal of Graphics Tools 3, 29-48 (1998)

11. Sidenbladh, H., Black, M.J., Fleet, D.J.: Stochastic tracking of 3D human figures using 2D image motion. In: Vernon, D. (ed.) ECCV 2000. LNCS, vol. 1843, pp. 702-718. Springer, Heidelberg (2000)

12. Hartley, R., Zisserman, A.: Multiple View Geometry in Computer Visio., 2nd edn. Cambridge University Press, Cambridge (2004)

13. Thrun, S., Burgard, W., Fox, D.: Probabilistic Robotics. MIT Press, Cambridge (2005)

14. Tomasi, C., Kanade, T.: Shape and motion from image streams under orthography: a factorization method. International Journal of Computer Vision 9, 137-154 (1992)

15. Quan, L.: Self-calibration of an Affine Camera from Multiple Views. International Journal of Computer Vision 19, 93-105 (1994)

16. de Boor, C.: A Practical Guide to Splines. Springer, Heidelberg (1978)

17. Coleman, T.F., Li, Y.: An Interior, Trust Region Approach for Nonlinear Minimization Subject to Bounds. SIAM Journal on Optimization 6, 418-445 (1996)

18. Coleman, T.F., Li, Y.: On the Convergence of Reflective Newton Methods for Large-Scale Nonlinear Minimization Subject to Bounds. Mathematical Programming 67(2), 189-224 (1994)

19. Dennis Jr., J.E.: Nonlinear Least-Squares. In: Jacobs, D. (ed.) State of the Art in Numerical Analysis, pp. 269-312. Academic Press, London (1977) 